Recepción: 29/06/2018

Evaluación: 08/10/2018

Aprobación: 21/10/2018

Artículo de Investigación-Científica

DOI: https://doi.org/10.22267/rhec.192222.53

\title{
El debate Currie-Daza y la institucionalización de la enseñanza de la economía en la Universidad Nacional de Colombia ${ }^{1}$
}

\author{
Germán Raúl Chaparro² \\ Universidad Central, Colombia
}

\section{Resumen}

El proceso de institucionalización de los programas de economía en el país implica, entre otras cosas, definir su organización administrativa, establecer objetos de estudio y justificar su pertinencia. Este artículo tiene como objeto analizar el proceso de institucionalización de la enseñanza de la economía como programa académico de pregrado al interior de la Universidad Nacional de Colombia y, a partir del debate entre dos importantes directivos de los programas, a principios de los años sesenta del siglo XX, ellos son Álvaro Daza Roa y Lauchlin Currie, quienes defendieron dos visiones distintas, y prácticamente antagónicas, de la economía. El primero se refiere a la economía como un mecanismo que debe ser estudiado cuantitativamente y cuyo propósito es la ganancia privada; Currie, por su parte, concibe la economía como un organismo, que amerita un estudio principalmente cualitativo y que debe estar orientado a mejorar el bienestar material de la comunidad. Se espera que el documento contribuya a entender la importancia histórica de los debates en torno a la enseñanza de la economía.

Palabras clave: Enseñanza de la Economía; Enseñanza de las Ciencias Sociales; Enseñanza Superior; Universidad.

$1 \quad$ Este artículo se deriva de la investigación: Lógicas sociales de la moneda: un análisis multidisciplinar; Componente: La enseñanza de la economía política en América Latina, avalado por el Grupo de Investigación Ataralawaa Amaa, de la Universidad Central, Colombia.

2 Doctorando en Historia de la Universidad Nacional de Colombia, Colombia. Magister en Economía. Docente de la Universidad Central, Colombia. Grupos de investigación: Protección Social de la Universidad Nacional de Colombia; Ataralawaa Amaa de la Universidad Central, Colombia. Líneas de Investigación: Protección social; Historia del pensamiento económico; Tributación. Correo electrónico: rchaparrog@unal.edu.co. Código ORCID: https://orcid.org/0000-0002-6443-0021 


\title{
The Currie-Daza debate and the institutionalization of teaching Economics in the National University of Colombia
}

\begin{abstract}
The process of institutionalization of economic programs in the country involves, among other things, defining their administrative organization, establishing objects of study and justifying their relevance. The purpose of this article is to analyze the process of institutionalization of the teaching of economics as an undergraduate academic program within the National University of Colombia and, based on the debate between two important directors of the programs in the early sixties of the twentieth century, Álvaro Daza Roa and Lauchlin Currie, who defended two different, and practically antagonistic, views of economics. The first refers to the economy as a mechanism that must be studied quantitatively and whose purpose is private gain; Currie, on the other hand, conceives the economy as an organism that deserves a mainly qualitative study and that it must be oriented to improve the material well-being of the community. It is hoped that this document will contribute to an understanding of the historical importance of the debates surrounding the teaching of economics.
\end{abstract}

Keywords: Economics Teaching; Social Sciences Teaching; Higher Education; University. 


\section{O debate Currie-Daza e a institucionalização da educação econômica na Universidade Nacional da Colômbia}

\section{Resumo}

O processo de institucionalização de programas de economia no país implica, entre outras coisas, definir sua organização administrativa, estabelecer objetos de estudo e justificar sua relevância. O objetivo deste artigo é analisar o processo de institucionalização da educação da econômia como um programa acadêmico de graduação na Universidade Nacional da Colômbia e, com base no debate entre dois importantes executivos dos programas, no início dos anos sessenta do século XX, são Álvaro Daza Roa e Lauchlin Currie, que defenderam duas visões diferentes e praticamente antagônicas da economia. O primeiro refere-se à economia como um mecanismo que deve ser estudado quantitativamente e cujo objetivo é o ganho privado; Currie, por outro lado, concebe a economia como um organismo, que merece um estudo principalmente qualitativo e deve ser orientado para melhorar o bem-estar material da comunidade. Espera-se que o documento contribua para entender a importância histórica dos debates em torno do ensino da economia.

Palavras-chave: Ensino da economia; Ensino de Ciências Sociais; Ensino superior, Universidade. 


\section{Introducción}

A lo largo de su Historia, la Universidad Nacional de Colombia se ha caracterizado por la promoción del espíritu crítico y el esfuerzo de orientar su actividad académica a la comprensión de los problemas propios de la sociedad colombiana; sin embargo, al recorrido de la Universidad y sus Facultades lo ha marcado una diversidad de interpretaciones y debates en torno a la organización institucional, los cambios curriculares, los contenidos de enseñanza, el perfil del egresado y los vínculos de la actividad académica con el acontecer nacional. Estos debates han sido recurrentes en distintos momentos de la Historia, con distintos protagonistas y en la diversidad de espacios académicos que componen su estructura organizacional. Este fue el caso del proceso de institucionalización del Programa de Economía.

Este artículo tiene como objeto analizar el proceso de institucionalización de la enseñanza de la Economía como programa académico de pregrado, en la Universidad Nacional de Colombia, a partir del debate entre dos importantes directivos de los programas, a principios de los años sesenta, del siglo XX, Álvaro Daza Roa y Lauchlin Currie, quienes controvirtieron a propósito de la enseñanza de la Economía, la organización y pertinencia del Programa de Economía.

Las controversias en torno a la enseñanza de la Economía parecen perdurar en el tiempo y permiten constatar que el proceso de construcción institucional no es acumulativo, pues se sujeta a vaivenes, rupturas, anunciadas por polémicas y debates, que se tratan de resolver por medio de la discusión académica razonada. Recordar estos episodios permite ponderar los logros institucionales, en términos de trayectoria académica y autonomía conseguida.

El debate entre las concepciones de Daza y Currie es pertinente, por cuanto subraya el hecho de que varios factores influyen en la determinación de la institucionalización de la enseñanza de la Economía. Los factores aludidos corresponden a la concepción de lo que se considere como Economía, a las necesidades del país, como, también, a las convicciones propias de los agentes reformistas referentes a qué es el conocimiento, cuáles son sus formas de adquisición, producción y propagación; incluso sobre temas como las modas analíticas del momento. 
La metodología de este artículo supuso la revisión bibliográfica de un conjunto de artículos y capítulos de libros sobre el tema. En particular, se analizaron artículos de los mismos protagonistas del debate, sobre su concepción de lo que debería ser y lo que constituyó la estructuración y funcionamiento de un Programa de Economía. Análogamente, se tomó en cuenta material referente al marco institucional de la época, como, también, material correspondiente a la coyuntura concomitante. Por esto, se considera que el material revisado y analizado contribuye a exponer, en términos sencillos, pero esclarecedores, la etapa histórica analizada.

El artículo se divide en cuatro partes, cuya introducción es la primera; la segunda parte alude al planteamiento del problema; la tercera expone los resultados del estudio del debate y se subdivide en tres apartados: en el primero, se describen algunos hitos del proceso de institucionalización de los Programas de Economía a mediados del siglo XX, en particular del Programa de Economía en la Universidad Nacional de Colombia; en el segundo, se presenta el debate planteado entre Álvaro Daza Roa y Lauchlin Currie en torno a la enseñanza de la Economía y, en el tercer apartado, se presenta un análisis del debate. Por último, se presentan las conclusiones.

La institucionalización de los programas académicos es un proceso complejo, altamente relevante, como quiera que su estructuración y desarrollo determinan decisivamente la formación del capital humano en una sociedad. En vista de este hecho, el examen del proceso de institucionalización permite identificar los factores que inciden en su estructuración, así como la respectiva interacción. En el caso de la institucionalización de la enseñanza de la Economía en la Universidad Nacional de Colombia, el debate Currie-Daza representa un ejemplo pertinente para evidenciar los elementos clave que incidieron en el proceso de estructuración de un programa disciplinar, todo lo cual redunda en una valoración de los logros institucionales.

Así las cosas, el análisis de este debate permite ver cómo el proceso de estructuración del Programa de Economía de la Universidad Nacional fue el resultado de la conjunción de muchos factores y que, pese a su gran cantidad, se pudo identificar un grupo de elementos clave en ese proceso. En particular, se puede adelantar que la estructuración se relacionó directamente con aspectos institucionales, humanos y coyunturales. Los aspectos institucionales tuvieron que ver con liderazgo, capacidad y entorno institucional de la misma Universidad. Los aspectos humanos se relacionaron con asuntos referentes a la formación académica, experiencia de vida, estatus social (inclusive personalidad) de los encargados. 
Paralelamente, la coyuntura, también, ayudó a determinar la estructura y práctica institucional; por ejemplo, en ese periodo, la concepción cepalina del desarrollo se impuso, lo cual representó un referente conceptual del desarrollo, como, también, la atención a determinadas variantes del análisis keynesiano.

\section{La institucionalización de la profesión de economista en Colombia}

Esta sección contiene los principales resultados referentes al examen del debate Currie-Daza, efectuado con ocasión de la institucionalización de la profesión de economista en la Universidad Nacional de Colombia, correspondiente a la década de 1960. Así las cosas, esta sección se divide en dos partes: una primera, correspondiente a la institucionalización; allí se exponen los antecedentes de lo que fue la instauración del estudio de la Economía en tanto profesionalización; la creación de entidades antecesoras dentro de la misma Universidad Nacional, de la Facultad de Economía; y la creación propiamente dicha de la Facultad de Economía de la Universidad Nacional. La segunda parte de la exposición de resultados expone el debate Daza-Currie. Por último, el tercer apartado de esta sección presenta un análisis del debate.

Los primeros programas académicos de Economía en Colombia aparecieron en los años cuarenta del siglo XX. Sin embargo, hay antecedentes importantes en las décadas anteriores, que vale la pena reseñar, aunque no existe evidencia histórica concluyente para establecer cuál fue la primera institución educativa en ofrecer un programa formal conducente al título de economista (Montenegro, 2017). El primero de ellos es la creación de Escuelas tecnológicas, a partir del Decreto 140 de 1905, que da vida a la Escuela Nacional de Comercio, en la ciudad de Bogotá, cuyo primer rector fue Víctor Mallarino (Rivera, 2002). ${ }^{3}$ Una segunda experiencia importante la ha señalado Carol Villamil Ardila, quien ase-

3 La escuela expedía títulos de idoneidad comercial a quien aprobara con éxito el Plan de estudios, que tenía énfasis contable y en el que se encuentra una asignatura de Economía política: el Plan de estudios se estableció mediante el Decreto 187 de 1905: "En la Escuela de Comercio de la capital de la República se dictarán las siguientes enseñanzas:

Religión, Castellano (primero y segundo cursos), Aritmética (primero y segundo cursos), Historia Patria y Geografía de Colombia, Geografía Comercial y Cosmografía, Francés (primero y segundo cursos), Inglés (primero y segundo cursos), Historia Universal, Física y Química elementales, Contabilidad mercantil, Práctica mercantil, Liquidación de facturas, Cuentas corrientes, Negocios de importación y exportación, Contabilidad oficial, Código de Comercio y Legislación Fiscal, y Economía Política. Habrá, además, un taller para trabajos en madera" (Decreto 187 de 1905, Art. 1). 
gura que los estudios económicos en la Universidad Nacional iniciaron en 1928, con la creación de la Facultad de Ciencias Económicas y Sociales, como órgano dependiente de la Facultad de Derecho (Decreto ejecutivo del 21 de marzo de 1928). Se trataba de una Facultad de estudios de especialización, que otorgaba el título de Doctor en ciencias económicas y sociales y en la que, en el primer año de funcionamiento, se habrían matriculado 40 abogados, la mayoría de ellos egresados de la misma universidad ${ }^{4}$ (Villamil Ardila, 2001, 80, n. 105)

Un tercer antecedente importante es la creación, en 1931, de la Facultad de Ciencias Económicas y Jurídicas, en la Pontificia Universidad Javeriana, bajo la influencia de Esteban Jaramillo y Mariano Ospina Pérez, que empezó a otorgar el título de abogado-economista (Mayor y Tejeiro, 1993, 204). El siguiente antecedente en el proceso de institucionalización de la enseñanza de la Economía y de otras disciplinas lo ha destacado Salomón Kalmanovitz: se trata de la fundación, en 1936, de la Escuela Normal Superior que, con su actitud científica en el análisis de los problemas sociales, permitió el desarrollo de una pedagogía liberal y las primeras interpretaciones sistemáticas de la realidad colombiana. Allí iniciaron su carrera docente Antonio García y Rudolf Hommes (padre), este último traído del Instituto Karl Marx, de Berlín, quien enseñó Economía Política en forma sistemática. Algunos egresados de esta institución hicieron aportes relevantes para la institucionalización de las Ciencias Sociales; por ejemplo, Darío Mesa, en el impulso de la sociología; Virginia Gutiérrez, en el campo de la antropología; y Jaime Jaramillo Uribe, en la promoción de la nueva historia (Kalmanovitz, 1993, 19).

En los años cuarenta, del siglo XX, aparecen de manera formal los programas de Economía a partir de la institucionalización de Institutos, Escuelas y Facultades, en instituciones educativas. En 1943, la Universidad Pontificia Bolivariana crea un Instituto de Economía y Comercio, en el que se ofrecieron estudios (Rhenals Monterrosa, 2003), y el Gimnasio Moderno funda la Escuela de Administración Industrial y Comercial que, luego, se fusionó con la Universidad de los Andes (Mayor y Tejeiro, 1003, 208). Al año inmediatamente siguiente, se

4 De este programa solo existen las primeras actas e informes del rector de la Facultad de Derecho, por lo que se supone que suspendió actividades a comienzos de la década de 1930 (Villamil Ardila, 2001, p. 90, n. 235). Se trataba de un programa de dos años, que incluía cursos de especialización en: Hacienda pública, Legislación comparada; Economía política e industrial; Legislación obrera, Código del trabajo, Cuestión social; Derecho administrativo; y Derecho mercantil, Legislación bancaria, Instrumentos negociables, Seguros, Sociedades, etc. 
presentaron dos proyectos de creación de unidades académicas en instituciones públicas: el del Instituto de Ciencias Económicas, de la Universidad Nacional de Colombia, y el de la Escuela de Ciencias Económicas, de la Universidad de Antioquia (Rhenals Monterrosa, 2003).

En cualquier caso, es relevante indicar que la Facultad de Ciencias Económicas, de la Universidad Nacional de Colombia, se organizó en 1952, momento en que la influencia política proviene del Partido Conservador. Doce años después, Lauchlin Currie cuestionó la pertinencia del enfoque de ese proyecto educativo.

\subsection{La creación del Instituto de Ciencias Económicas, en la Universidad Nacional de Colombia}

La matriz de la profesionalización de la Economía tradicionalmente proviene de la ingeniería o el derecho. En el caso de la Universidad Nacional, la matriz fue la Facultad de Derecho, lo cual explica que abogados de esta institución, como Carlos Lleras Restrepo, Antonio García y Luis Ospina Vásquez, incursionaran exitosamente en el campo de la Economía y expresaran la necesidad de crear programas académicos especializados en Economía, programas en los cuales, a la postre, serían docentes.

Antonio García Nossa, con el apoyo de Gerardo Molina, rector de la Universidad, inicia el proyecto de creación del Instituto de Ciencias Económicas, ${ }^{5}$ como dependencia anexa de la Facultad de Derecho, con el objetivo de crear una especialización en Ciencias Económicas para profesionales de distintas disciplinas. El Instituto enfatizaba en el estudio sistemático de "los problemas fundamentales de la economía pública" (Acuerdo 56, de 1945, Art. 1). En este proyecto, la enseñanza de la Economía se basaba en una visión social y política definida: la búsqueda de una sociedad más justa y más democrática. Además, se reconocía a la Economía como una disciplina independiente, con un cuerpo de conocimiento propio, en un contexto en el que, a la sazón, no había en el país una tradición académica de estudios en Ciencias Económicas en el ámbito profesional. A propósito, los profesores Antonio Hernández y Beethoven Herrera desta-

5 La creación Instituto la aprobó el Consejo Superior mediante el Acuerdo 56, del 13 abril de 1945, Acta 15 (Rivera, 2002). 
can, de Antonio García, su concepción de "La economía como ciencia social, el compromiso del trabajo académico con la realidad para encontrar allí los temas de estudio y trabajar en función de producir respuestas posibles a las necesidades del país" $(2002,20)$.

En general, los programas creados en la década de 1940 tuvieron una orientación en aspectos jurídicos, administrativos y contables (Rodríguez Salazar y Arévalo Hernández, 1994, p. 238). En el transcurso de esa década, el oficio de economista empezó a convertirse en una actividad de "tiempo completo", producto del incremento de la demanda de especialistas por parte de instituciones como el Banco de la República y la Contraloría General de la Nación. El Banco había creado, desde 1928, la "oficina de estadística" que, en 1945, se transformó en "oficina de investigaciones económicas". Por su parte, la Contraloría General de la Nación emprendió la investigación que produjo el censo poblacional de 1938, el censo industrial de 1945 y publicaciones periódicas, como el Anuario General de Estadística y la revista Economía y Estadística, que fueron la base del Boletín Mensual de Estadistica, que empezó a publicar el DANE, a partir de 1955 (Mayor y Tejeiro, 1993, 208).

En la primera mitad de la década de 1950, la presencia en el país de misiones internacionales, como la Misión del Banco Internacional de Reconstrucción y Fomento, de 1949, encabezada por Lauchlin Currie; la misión de la Cepal, de 1954; y la misión de economía y humanismo del padre Lebret, en 1955, fueron importantes en el proceso de institucionalización de la Economía (Kalmanovitz, 1993) y, también, en la interpretación de la Historia económica colombiana (Rodríguez Salazar y Arévalo Hernández, 1994, 237).

\subsection{La creación de la Facultad de Economía}

Al iniciar los años cincuenta del siglo XX, el Instituto de Ciencias Económicas fue "purgado por la administración conservadora" (Kalmanovitz, 1993, p. 29) y transformado en la Facultad de Economía, que condujeron los decanos: Eduardo Suárez Glasser y Álvaro Daza Roa, y el director del Departamento de Economía, Jaime González Santos, con una orientación casi que antagónica respecto a su inspiración original. En la recién creada Facultad, se privilegió una concepción de la Economía como técnica. Kalmanovitz señala que, con el cambio del Plan de estudios, "se dejó de estudiar la economía pura, se introdujo la 
economía del justo precio, muchas finanzas y contabilidades, administración de empresas y una parte considerable al estudio del derecho", con lo que se empezaron a producir "los profesionales: administrador-contador, abogado-economista" $(1993,29)$. Antonio Hernández y Beethoven Herrera señalan que, en esos tiempos: "Las inquietudes estudiantiles y los debates sobre la realidad nacional desde una perspectiva crítica tuvieron que refugiarse en las cafeterías y construir sus propios espacios de difusión" $(2002,18)$.

\section{El debate Daza-Currie sobre la orientación de la enseñanza de la Economía en Colombia}

En 1964, se produjo, en la Universidad Nacional, un debate sobre la pertinencia de reorganizar institucionalmente las Facultades. En este contexto, Álvaro Daza Roa y Lauchlin Currie presentaron sus reflexiones sobre lo que ambos describieron como "un examen de conciencia" en torno a la enseñanza de la Economía, en Colombia (Daza Roa, 1965, 1; Currie, 1965, 10).

El 30 de octubre de 1964, Álvaro Daza Roa pronunció un discurso con ocasión de la celebración del Día del Economista, en el que, al parecer, presentaba sus descargos ante un ambiente de crítica que gravitaba en ese momento en torno a la formación profesional que se impartía en la Facultad de Economía. El decano comenzó su defensa con excusas sobre las dificultades impuestas a los jóvenes economistas, a quienes "se les exigen realizaciones casi imposibles de lograr" en un país con "el deseo de un desarrollo más acelerado, pero sin objetivos definidos; cambios sociales, pero sin criterios claros y precisos" (Daza Roa, 1965, p. 1), condiciones que justificarían la formación de economistas híbridos, es decir, con formación tanto en Economía pública como en Economía privada:

No es lo mismo el tratamiento que se le da a la economía del Estado que el de las empresas particulares, porque ello equivaldría a identificar las motivaciones públicas con las de los particulares. Esta diferenciación ha conducido a distinguir entre la economía pública y la economía privada y la necesidad de proporcionarle al economista, además de las herramientas generales de la ciencia, herramientas no «coincidentes», herramientas más especializadas que le sirvan para realizar su trabajo en forma adecuada y eficiente, según sus inclinaciones y sus orientaciones $(1965,7)$. 
El decano aseguraba que la división de la Economía en pública y privada no implicaba una confusión respecto a la formación de los administradores:

El economista, ya pertenezca a la orientación pública o privada, proporciona las pautas para el logro del mejor aprovechamiento de los recursos escasos según los objetivos que se predeterminen y el administrador, gobierna, cuida, rige y gestiona las actividades de la entidad o instituciones que estén a su cuidado $(1965,8)$.

Respecto al tema de la disyuntiva entre estudios generales y estudios especializados, Daza planteaba que:

Es encomiable que todas las personas tengan conocimientos generales de economía $[\ldots]$ pero no es encomiable que un estudiante que espera ser profesional de la economía no se dedique desde un comienzo a desentrañar los principios de su ciencia y a habilitarse a pensar en sus complejidades $(1965,4)$.

Desde la creación de la Facultad de Economía, en 1952, habría existido un compromiso con ofrecer al estudiante un conjunto de instrumentos de análisis cuantitativo, cuya importancia principal radica en desarrollar la capacidad para "pronosticar". Desde la perspectiva de Daza Roa, la Economía "debe ser considerada como una «caja de herramientas» y como un instrumento analítico y no como una «caja de dogmas» o de «generalizaciones literarias»" $(1965,6)$

La supremacía del análisis cuantitativo acrecienta la necesidad de formulaciones matemáticas para poder superar la etapa que Jevons denominaba de los «economistas literarios» y superar la etapa de las conclusiones introspectivas y las conclusiones de escritorio. Las hipótesis deben confrontarse con la realidad; y el economista, en nuestros países, está en la obligación de buscar estas comprobaciones. $(1965$, p. 5)

Y, finalmente, en cuanto a la organización de la Facultad y sus programas de estudio, planteaba la necesidad de "coordinar e integrar en una Facultad de Ciencias Económicas y Administrativas, los departamentos de Economía, de Administración, de Contaduría, de Estadística y Actuaría, junto con el Centro de Investigaciones Económicas, el Centro de Productividad y una Escuela de Graduados.” (Daza Roa, 1965, 9) 
Por su parte, el examen de conciencia propuesto por Lauchlin Currie ${ }^{6}$ se produjo en un escenario en que los grados académicos en Economía en el país crecían de manera aparentemente desproporcionada, para atender una moda, sin planificación ni coordinación, y sin ninguna, o muy poca, consideración sobre su papel en la vida nacional. Currie comenzaba su análisis con el reconocimiento de su inclinación por un tratamiento más cualitativo y conceptual, que cuantitativo y descriptivo, en la enseñanza de la Economía en Colombia:

Mi tipo de mente es de aquellas que se siente más a gusto con los conceptos que pueden expresarse en términos literarios, y esto puede viciar mi juicio, aunque confío que no. Con prejuicios o sin ellos, mi posición es la de que un país opulento puede darse el lujo de permitirle mucha libertad a sus profesores universitarios y científicos para seguir sus inclinaciones. En el caso de Colombia, sin embargo, donde las necesidades son apremiantes y los recursos son estrictamente limitados, yo insistiría en la prioridad de una preparación básica en teoría económica en los términos más sencillos y menos técnicos que fuera posible. Nuestra primera necesidad es la de que los estudiantes universitarios tengan alguna visión sobre el funcionamiento del sistema económico y aprendan, al menos en forma limitada, a aplicar los conceptos básicos a problemas reales. Para este objetivo limitado, yo sostendría que una presentación matemática constituiría un obstáculo en vez de una ayuda. La economía es una ciencia que trata de ciertos aspectos de la conducta humana. Se asemeja más a un organismo que a un mecanismo, es imprecisa y descansa sobre bases emocionales. El estudiante debe apreciar todo esto y no dejarse llevar a un mundo imaginario de aparente precisión matemática (Currie, La Enseñanza de la Economía en Colombia, 1965, 18-19).

Currie era partidario de reintegrar a la Economía dentro del conjunto de las Ciencias Sociales, evitar la tentación de pensar en las distintas disciplinas como compartimentos estancos y promover el diálogo interdisciplinar. Esto queda claro en la definición de Economía que propuso:

La economía es una de las ciencias sociales o de comportamiento que intenta formular generalizaciones válidas sobre aquellos aspectos de la conducta humana que se refieren primariamente a la producción y distribución de los bienes y servicios (Currie, La Enseñanza de la Economía en Colombia, 1965, 10).

Y agregaba:

$6 \quad$ Las reflexiones de Currie son producto de las visitas realizadas a las Facultades de Economía del país por encargo de la Asociación Colombiana de Universidades. 
la economía es la ciencia que se ocupa sobre todo del bienestar material y, de entre todas las ciencias, la que se ocupa en forma exclusiva de la organización de la producción y de la distribución de los bienes y servicios, no desde el punto de vista del individuo, sino de la comunidad (Currie, La Enseñanza de la Economía en Colombia, 1965, 29-30).

La apreciación de la Economía como una ciencia que se ocupa del bienestar material de la comunidad lo conduce a reconocer que un mismo campo de estudio se puede abordar por distintas razones; sin embargo, resulta impráctico tratar de cubrir con muchos detalles todos los temas comprendidos en la organización económica de la sociedad. Así, por un lado, se aplica el principio de la división del trabajo y las ventajas derivadas de la especialización, pero, por el otro, el exceso de especialización implica dejar de cubrir grandes áreas del campo, lo que conduciría "a la conocida y repetida definición del experto como la persona que sabe más y más de menos y menos" (Currie, La Enseñanza de la Economía en Colombia, 1965, 11).

Por eso, la necesidad de llegar a un acuerdo más o menos arbitrario "que permita el estudio intensivo de los elementos más esenciales del sistema económico desde el punto de vista de su funcionamiento general, a la vez que suministrar una ligera enseñanza sobre los elementos y desarrollos esenciales en campos relacionados con ella." De esta manera, Currie propone que, en la enseñanza de la Economía, al igual que en el campo de la medicina, antes de llegar a la concentración o especialización "es deseable que se logre la comprensión general del funcionamiento del sistema económico como un todo" (Currie, La Enseñanza de la Economía en Colombia, 1965, 11).

A la sazón, la distinción entre Economía y Administración de Negocios resultaba borrosa, pero la clave estaba, según Currie, en identificar las motivaciones que hacían parte del objetivo de estudio; de esta forma, complementaba la definición de Economía como una ciencia que:

Busca establecer generalizaciones, comprender el funcionamiento de los sistemas económicos ya sean éstos de libre iniciativa privada, socialistas o mixtos, y capacitar al hombre para moldear y rehacer su medio ambiente económico. La administración de negocios puede realizar en parte el mismo conocimiento, pero con el objetivo de ganancia privada $(1965,11)$. 
Así, se advierte que resulta perjudicial que una misma Facultad impartiera los dos programas y recomendaba su separación en dos campos de estudio. En este punto, la tendencia en Colombia había sido confundir las dos profesiones y encubrir la confusión con el uso de las expresiones "Economía pública" y "Economía privada":

No puede ser perjudicial y, en cambio, sí muy benéfico que los hombres de negocios, como miembros educados de la clase dirigente, posean un conocimiento no tan superficial del funcionamiento del sistema económico donde viven. Pero su interés primario debe ser naturalmente el de ganar dinero. Tal motivación, sin embargo, es fatal para un trabajo científico de primera clase (Currie, La Enseñanza de la Economía en Colombia, 1965, 12). ${ }^{7}$

También, señalaba la necesidad de distinguir entre la Economía como ciencia y la planeación económica como arte. En Colombia, a nivel de los ministerios y de las entidades regionales se ha llamado "planeación" a las actividades de evaluación de proyectos o programas, lo que poco tiene que ver con el campo general de la Economía:

La economía, como tal, se ocupa del análisis, comprensión y generalización. La planeación económica nacional se ocupa de la elección de metas, el diagnóstico de conjuntos especiales de condiciones y los modos y medios de alcanzar ciertos fines específicos. Para ello emplea herramientas del análisis económico, así como el conocimiento y juicios obtenidos de otras fuentes (Currie, La Enseñanza de la Economía en Colombia, 1965, 13).

En cuanto a la forma en que se debe enseñar Economía y su público objetivo, Currie era partidario de que la Economía fuera la parte de un cuerpo de conocimientos generales común a todas las disciplinas académicas. Toda persona ilustrada debería tener una comprensión suficiente del funcionamiento del sistema económico, que le permitiese distinguir lo necesario y deseable de lo demagógico. En este sentido,

para nuestra salvación nacional, las universidades tienen una responsabilidad importante que es la de suministrarle a todos o a una gran proporción de sus estudiantes una preparación elemental en economía, lo suficientemente buena

7 Para la enseñanza de la administración, Currie propuso seguir el sistema de la Escuela de Negocios de la Universidad de Harvard, que establecía un énfasis en los casos reales, donde la contribución de la Economía consiste en ofrecer una «cultura general», más que pretender que fuese una materia «práctica». 
como para capacitar a la clase dirigente para alcanzar una buena comprensión de nuestro sistema económico (Currie, La Enseñanza de la Economía en Colombia, 1965, 14-15).

De esta manera, Currie proponía la integración de las Facultades existentes, en una Facultad de Ciencias sociales o de comportamiento, y ofrecer una preparación profesional general, con una duración entre dos y cuatro años, que rompiera con el sistema rígido de Facultades separadas, y, luego de ello, ofrecer formación especializada para unos pocos, con énfasis en investigación y enseñanza (1965, 17 y 19), dentro de la cual se podría considerar la posibilidad de ofrecer una especialización en Economía matemática, opción que no dejaba de estar libre de riesgos:

Aún en planeación nacional, necesitamos tanto de la mente intuitiva que llega a efectuar juicios certeros en forma casi inconsciente como de los expertos en cuentas nacionales o en efectuar proyecciones sobre la base de datos estadísticos dudosos. Al insistir en una preparación matemática avanzada, existe el peligro de perder para la economía al primer tipo de mentalidad, lo cual difícilmente podemos darnos el lujo de hacer (Currie, La Enseñanza de la Economía en Colombia, $1965,19)$.

Currie destacaba que la prioridad debía ser la teoría, que consideraba era más útil para atender los problemas de un país desarrollado:

Las matemáticas y las técnicas estadísticas pueden constituir herramientas útiles de análisis para ciertos tipos de problemas [...] Pero los conceptos económicos básicos son mucho más útiles al tratar con la mayoría de problemas económicos de un país subdesarrollado (Currie, La Enseñanza de la Economía en Colombia, 1965, 37).

Planteaba, igualmente, que los desarrollos de la Economía matemática habían permitido presentar los conceptos básicos de la Economía en un lenguaje con "apariencia (por lo general falsa) de precisión. [...] De esta manera, se crea un escape del mundo confuso e insatisfactorio de las ciencias sociales" (Currie, La Enseñanza de la Economía en Colombia, 1965, 18).

Finalmente, Currie resultaba crítico de la importancia que, en Colombia, se otorgaba a los títulos, y del abuso del título de doctor. Por ello, consideraba como un riesgo la creación de grados y títulos de «economista», o incluso de «planificador», pues el título podría perder prestigio. Currie era partidario de que 
se reservase el término economista para quienes demostraran con su experiencia que verdaderamente estaban ejerciendo la profesión, ya fuera en la enseñanza o en la investigación (Currie, La Enseñanza de la Economía en Colombia, 1965, 21-23).

A pesar de las advertencias de Currie, con el Decreto 1297 de 1964, se autorizó a las universidades para conferir el título de economista como profesión, mientras que, paradójicamente, en el área de Ciencias Sociales se otorgaba el título de licenciado. Al interior de la Universidad Nacional, las discusiones sobre la organización de las Facultades, en particular sobre la enseñanza de la Economía, condujeron a que la Facultad de Economía se convirtiera en un Departamento de la Facultad de Ciencias Humanas.

\section{Análisis del debate: algunos elementos}

En el caso de las posiciones de los protagonistas aquí examinados, según Lauchlin Currie, la Economía es la ciencia que se ocupa principalmente del bienestar material de la sociedad. ${ }^{8}$ En este sentido, busca establecer generalizaciones, comprender el funcionamiento de los sistemas económicos, así como capacitar al hombre para formar y rehacer su medio económico. En general, su preeminencia está en el abordaje y resolución de determinados problemas sociales, mediante el suministro de perspectivas analíticas generales de alto nivel de abstracción, en oposición al criterio según el cual el estudio de la Economía se reduce a la obtención de un conocimiento instrumental cristalizado en la adquisición de una caja de herramientas y de unas técnicas determinadas. Según Currie, la economía se ocupa del análisis, comprensión y generalización, sentido por el cual los agentes responsables de planear la Educación Superior deben distinguir claramente entre la ciencia de la economía, la administración de negocios y las habilidades y requisitos especiales que demanda la política económica, como quiera que "Tratar de preparar simultáneamente a los estudiantes en todos estos campos diversos es cortejar el desastre y el descrédito completo, tanto de la economía como de la planeación" (Currie, La Enseñanza de la Economía en Colombia, 1965).

\footnotetext{
$8 \quad$ Nótese que, desde la perspectiva del autor, al existir solo una ciencia de la Economía, la distinción entre Economía pública y Economía privada resulta improcedente.
} 
Por su parte, según Álvaro Daza, la formación básica en Economía se representaba en la obtención y uso de instrumentos analíticos plasmados en la enseñanza y aplicación de modelos económicos, como se presentaban en los manuales de economía ortodoxa. Según su concepción de la Economía, un programa en esta área debía propender por dotar al futuro graduado de instrumentos analíticos y de una caja de herramientas que le permitieran resolver problemas prácticos, reales. Empero, dada su visión de formar economistas para la Economía pública y para la Economía privada, en tanto funcionarios medios y hombres de empresa, respectivamente, poco o nada señalaba respecto a la necesidad de formar economistas investigadores y técnicos de primer nivel, co-determinadores de políticas económicas o sus críticos.

Respecto al desarrollo teórico, cada una de las concepciones consideradas tenía una posición. Currie era un seguidor del keynesianismo, si bien bastante prudente respecto a la intervención del Estado, que consideraba que el problema de desarrollo de la economía colombiana lo constituía una insuficiente demanda efectiva consecuente con un mayor y mejor aprovechamiento de las correspondientes economías de escala derivadas de una producción a nivel industrial. Para el caso, resultaba imperativo realizar una redistribución de los recursos humanos y una adecuación técnica; por ejemplo, orientar la fuerza de trabajo rural al dominio urbano, en calidad de trabajadores no calificados en sectores como la construcción, o tecnificar el sector agropecuario para elevar las tasas de productividad. En este sentido, era partidario de una expansión del gasto financiado; en el ámbito urbano, dicha financiación no suponía un incremento de impuestos, sino su mejor recolección y aplicación; en el ámbito rural, una imposición efectiva a las tierras improductivas (Currie, 1961).

Por su parte, Álvaro Daza Roa era un profesor postgraduado de la Universidad de Vanderbilt, en los Estados Unidos, decano de la Facultad de Economía de la Universidad Nacional a inicios de los años sesenta, y profesor de varias asignaturas allí. Más que un seguidor de una escuela específica de la ortodoxia económica, planteaba que el futuro economista debía adquirir las herramientas analíticas provistas por el paradigma ortodoxo, en sus principales versiones. Exponía que el economista debía ser un técnico, solucionador de problemas reales mediante el análisis y uso de modelos cuantificables. 
La insistencia de Daza en el rol de técnico cuantitativo que debería desempeñar el economista se debía a su posición respecto a la situación social, económica y cultural del país. Al respecto, expresaba que la situación de carencia experimentada por la sociedad colombiana y la concomitante urgencia de responder a esa condición, impedían la preparación de economistas investigadores, sobremanera la producción de conocimiento original, de calidad y utilidad equiparable a la de los centros académicos de vanguardia. Por lo tanto, la preparación ofrecida debía ser práctica, orientada a la solución de problemas reales, concretos, y no a la elaboración de "generalizaciones literarias" y de "conclusiones de escritorio". En su opinión, la formación impartida debía superar la formación de economistas literarios. En consecuencia, los contenidos suministrados tenían que ser prácticos, reales y específicos, de tal suerte que los problemas usuales de la Economía pública, por caso, la elaboración de conceptos técnicos referidos a resultados o impactos de intervenciones públicas, o de la Economía privada, tales como la valoración de la situación gremial de determinado sector productivo, se resolvieran mediante el uso de la caja de herramientas y de las técnicas adquiridas en la academia. Correlativamente, consideraba que el economista no era una especie de "todero" formado para resolver todo tipo de problemas, por ejemplo, la reducción de la desigualdad, la precariedad de las condiciones de vida del campesinado, o el desequilibrio social y económico entre el campo y la ciudad. El profesional estaba, eso sí, para resolver una clase específica de problemas, según su ámbito de aplicación, bien fuera que se tratara de la Economía pública o de la Economía privada.

De otro lado, respecto a los Planes de estudio de la Universidad Nacional, cada uno de los protagonistas aquí examinados tenía una postura bastante disímil, rayana en el antagonismo. La dirección de Daza se caracterizó por la instauración de un gran número de asignaturas, distribuidas en distintas materias de orden práctico ("útiles", referentes a matemáticas, estadísticas y contabilidades), plasmadas en un exceso de clases magistrales, con ejercicios mecánicos, adiestramiento que, en todo caso, restaba tiempo para la subsunción en actividades analíticas y actividades como la lectura. En 1960, el Programa de economía se componía de dos ciclos: uno general (de tres años) y otro especializado (de dos años), según el tipo de especialización que quisiera tomar el estudiante (esto es, bien fuera Economía pública o Economía privada). El Ciclo general comprendía las áreas de análisis cuantitativo e información estadística, y el área de teoría económica (micro y macroeconomía, teoría monetaria, desarrollo económico, etc.). El Ciclo de especialización comprendía estas opciones: 1) economía pú- 
blica, (comprendía asignaturas de administración pública, presupuesto nacional y teoría del desarrollo, entre otras), 2) economía privada (que incluía materias de contabilidad y administración de empresas), o 3 ) estudios en estadística (orientada a un fortalecimiento cuantitativo, básicamente en econometría) (Sarmiento Aranzales, 2002).

Como se señaló, según Currie, la Economía es una ciencia social que pretende formular generalizaciones válidas sobre determinados aspectos de la conducta humana referentes a la producción y distribución de los bienes y servicios. Claramente, por ser el comportamiento humano el resultado de la interacción de múltiples factores, y por ser la Economía una ciencia que estudia determinadas facetas de comportamiento humano, su estudio no se puede hacer de forma aislada, independiente de las otras disciplinas que examinan otras facetas del ser humano. Es claro, las divisiones que se han hecho para abordar los asuntos humanos se han elaborado por cuestiones de conveniencia analítica, para facilitar su tratamiento, lo cual no quiere decir que los asuntos humanos no se interrelacionasen (por ejemplo, el comportamiento económico de los individuos no es ajeno a las emociones, a la cultura o a la condición social). Entonces, de conformidad con el planteamiento de Currie acerca de que la Economía es una ciencia social, en 1966, la Facultad de Economía de la Universidad Nacional experimentaría una nueva orientación, esta vez en calidad de Departamento de Economía de la Facultad de Sociología, razón por la cual se verificaría una diversidad temática en cuanto al programa curricular, como también en el énfasis y enfoque. ${ }^{9}$

Frente a la dirección de Daza, se destacan algunas características del Programa de Economía. A la sazón, existían problemas de diverso orden, referentes al profesorado, que lo componían en buena medida abogados y hombres prácti-

$9 \quad$ En contraste con la posición de Daza, según Currie no había dos Economías: "solamente existe una ciencia de la economía. La economía 'privada' es tan solo un eufemismo para la administración empresarial o de negocios" (Currie, 1965, p. 131), sentido por el cual no se debe confundir a la opinión general. Además, Currie enfatizaba en que el estudio de la Economía no produce economistas con los mismos puntos de vista u opiniones, tal como puede ocurrir con profesionales de disciplinas técnicas. En este sentido, la planificación o planeación económica no se puede aprender de forma mecánica, por lo cual Currie era de la opinión que al intentar “enseñar 'planeación' en una etapa temprana de la preparación del economista, se corre el peligro de supersimplificar lo que es tal vez el ramo más difícil en el cual pueda trabajar un economista, y de crear rigideces mentales. Lo que se requiere es estar alerta, tener originalidad y agilidad mental combinadas con una preparación a fondo en teoría económica" (Currie, 1965, p. 131). 
cos. Además, no existían profesores de tiempo completo proclives a mejorar la docencia como tampoco a implicar la investigación en ella. Ante todo, se debe subrayar que muchas de las asignaturas "estaban entonces a cargo de parlamentarios y columnistas con escasa formación en el análisis económico riguroso. A lo que es preciso añadir la ninguna preocupación del pensum por las causas del atraso económico y social de la nación colombiana y menos aún por inquirir acerca de los factores que hacían posible su superación." (Hernández Gamarra, 2002, p. 417). En suma, el programa impartido era bastante débil. De un lado, adolecía de problemas misionales relacionados con la docencia y la no implicación de la investigación en ella, por ejemplo, por no contar con una planta de docentes debidamente motivados y preparados, incluidos de tiempo completo. De otro lado, el programa resultaba impertinente en grado significativo, puesto que solo se dictaban asignaturas técnicas, pero no cursos que desarrollaran competencias analíticas, ni materias que abordaran debidamente los problemas del país.

Como marco de referencia, se debe señalar que el periodo examinado se caracteriza por dos elementos: 1) durante los años 60's se estableció el cepalismo como estrategia de desarrollo; 2) durante ese periodo se verificó una agitación política que, en todo caso, incidió en la forma de considerar los problemas sociales, en particular los económicos. ${ }^{10}$ Respecto al enfoque cepalista para el desarrollo de Colombia, durante el periodo examinado, no hubo debate alguno de gran peso académico; por ejemplo, una posición de la academia frente a la estrategia de desarrollo plasmada en la industrialización y la sustitución de importaciones. En relación con el segundo punto, los temas de debate que ocupaban tiempo, algunos de los estudiantes referían a las tensiones políticas del momento, relacionadas con la violencia política. En una palabra, en la primera mitad de los 60's, se dedicó mucho tiempo a la recepción de conocimientos vía memorización y práctica mecánica de ejercicios, y poco espacio para el verdadero adiestramiento del pensamiento afín a una comprensión de la complejidad propia del sistema económico. Entrada la segunda mitad de esta década, se verificaría una oferta más copiosa de temas curriculares, así como mayor diversidad de enfoque, sentido por el cual se puede afirmar que las limitaciones de pertinencia verificadas en la primera mitad se relajaron.

10 Según Currie, tres eran los problemas más acuciosos de la sociedad colombiana: bajos niveles de ingresos, elevados índices de natalidad y, sobre todo, la violencia política exacerbada a finales de la década de 1950, debido al asesinato de Jorge Eliécer Gaitán. 


\section{Conclusiones}

Al proceso de institucionalización de la carrera de Economía al interior de la Universidad Nacional de Colombia lo marcaron los debates en torno a la organización de las Facultades y la enseñanza de la Economía; es evidente el papel de las controversias en la definición de la trayectoria seguida por la Universidad y sus programas de estudio, así como, también, la importancia de los actores que, por su capacidad académica, influencia política y carisma, han podido incidir en la toma de decisiones que afectaban el devenir del proyecto colectivo.

El reto de formar profesionales idóneos, capaces de aplicar sus conocimientos a los problemas del país, ha tropezado con problemas recurrentes, como la escasez de recursos financieros y humanos, la poca comprensión de la necesidad de formar profesionales competentes, la presión oficial para definir orientaciones académicas y la baja valoración por parte de sectores de la sociedad respecto a la importancia de la educación. De allí la importancia de valorar el hecho de que la Universidad Nacional ha logrado consolidar, a través de su historia, niveles importantes de autonomía universitaria, de promoción de un espíritu crítico, de compromiso con la verdad y de esfuerzo en la búsqueda de soluciones para las necesidades del país.

Lauchlin Currie defendió un enfoque cualitativo y conceptual en el estudio de la Economía como un organismo vivo, cambiante, impreciso y emocional, que debería estudiarse a partir de generalizaciones teóricas y con énfasis en el estudio de la conducta humana, con el fin de mejorar la comprensión del sistema económico. La motivación del estudio de la Economía es su rasgo distintivo, que consiste en propiciar la transformación de las relaciones económicas de producción y distribución con el objetivo de lograr mejoras continuas en el bienestar material de la comunidad.

La visión de Currie contrasta con el enfoque mecanicista que promovía Álvaro Daza Roa, defensor de un enfoque cuantitativo y descriptivo, consistente en dotar al estudiante de instrumentos analíticos, una caja de herramientas, con la que estudia, por un lado, la asignación eficiente de recursos en contextos de escasez, que se denominó Economía pública; y, por otro lado, la Economía privada; es decir, la administración y gestión de intereses particulares, cuya motivación es la ganancia privada. 


\section{Referencias}

Currie, Lauchlin. Operación Colombia: Un programa nacional de desarrollo económico y social. Bogotá: Sociedad Colombiana de Economistas, 1961.

Currie, Lauchlin. La enseñanza de la Economía en Colombia. Bogotá: Ediciones Tercer Mundo, 1965.

Daza Roa, Álvaro. La Enseñanza de las Ciencias Económicas en la Universidad Nacional. Bogotá: Asociación Colombiana de Universidades y Fondo Universitario Nacional, 1965.

Hernández Gamarra, Antonio. "Alberto Corchuelo: sus inicios como economista”. En: Búsquedas y logros desde la academia, editado por Hernández Gamarra, Antonio y Herrera Valencia, Beethoven. Bogotá: Universidad Nacional de Colombia, 2002. 414-426.

Hernández Gamarra, Antonio, y Herrera Valencia, Beethoven. "Medio siglo de búsqueda de la razón por la crítica". En: Búsquedas y logros desde la academia, editado por Hernández Gamarra, Antonio y Herrera Valencia, Beethoven. Bogotá: Universidad Nacional de Colombia, 2002. 13-25.

Kalmanovitz, Salomón. "Notas para una historia de las teorías económicas en Colombia". En: Historia social de la ciencia. Ciencias Sociales. Vol. 9, editado por Vasco, Carlos Eduardo et al. Bogotá: Instituto colombiano para el desarrollo de las ciencias y la tecnología Francisco José de Caldas y Colciencias, 1993. 13-61.

Mayor, Alberto y Tejeiro, Clemencia. "La profesión de economista en Colombia: Entre el autodidactismo y el entrenamiento académico". En: Cambio técnico, empleo y trabajo en Colombia. Aportes a los estudios laborales en el VIII Congreso de Sociología, editado por Dombios, Rainer y López, Carmen Marina. Bogotá: FESCOL, 1993. 199-222.

Montenegro, Álvaro. "Los albores de la economía en Colombia". Revista de Economía del Rosario. Vol. 20. No. 2 (2017): 307-324. 
Rhenals Monterrosa, Remberto. De la perplejidad a la explicación: Crónicas de la Economía, su enseñanza y aplicación. Medellín: Universidad de Antioquia, 2003.

Rivera, M. (2002). "Institucionalización de los estudios económicos en la Universidad Nacional de Colombia, 1945-1952”. En: Búsquedas y logros desde la academia, editado por Hernández Gamarra, Antonio y Herrera Valencia, Beethoven. Bogotá: Universidad Nacional de Colombia, 2002. 22-50.

Rodríguez Salazar, O., \& Arévalo Hernández, D. "La historiografía económica colombiana del siglo XIX”. En: La historia al final del milenio: Ensayos de historiografía colombiana y latinoamericana, editado por B. Tovar Zambrano. Vol. 2. Bogotá: Universidad Nacional de Colombia. 1994. 187-250.

Sarmiento Aranzales, Alfredo. (2002). "La Facultad de Ciencias Económicas de la Universidad Nacional de Colombia: 1958-1961”. En: Búsquedas y logros desde la academia, editado por Hernández Gamarra, Antonio y Herrera Valencia, Beethoven. Bogotá: Universidad Nacional de Colombia, 2002. 401-407.

Villamil Ardila, Carlos. (2001). Aproximación histórica a la Facultad de Derecho, Ciencias Políticas y Sociales de la Universidad Nacional de Colombia. Bogotá: Universidad Nacional de Colombia, Facultad de Derecho, Ciencias Políticas y Sociales. 